\title{
Molecular detection of High Risk Human Papilloma Viruses (HR-HPV 16 and 18) among patients with esophageal cancer
}

\begin{abstract}
Background: The incidence of esophageal cancer continues to rise in Sudan. High risk Human papilloma virus (HR-HPV) has been postulated to play a major etiological role in the development of esophageal carcinoma. The aim of the present study was to identify HR-HPV 16 \& 18 among Sudanese patients with esophageal cancer.
\end{abstract}

\begin{abstract}
Methodology: This was study retrospectively examined 100 esophageal cancer tissues obtained from archived formalin fixed paraffin wax embedded tissue's blocks obtained from several histopathology laboratory in Khartoum State, Sudan.
\end{abstract}

Results: Molecular detection of HR-HPV 16 was revealed in $25(24.5 \%)$ and couldn't be evident in $77(74.5 \%)$ of the study subjects, hence, none of the cases was identified with HR-HPV18. Of the 57 males with esophageal cancer, 17/57 (29.8\%) were identified with HR-HPV16 infection, whereas, of the 45 females with esophageal cancer, $8 / 45(17.8 \%)$ were found positive for HR-HPV 16

Conclusion: HR-HPV subtype 16 is significantly contributed to the etiology of esophageal cancer in Sudan. Further studies are required to determine the burden of other HR-HPV subtypes in esophageal cancer.

Keywords: Esophageal Cancer, Sudan, HR-HPV16, HR-HPV18
Volume 5 Issue 3 - 2017

\author{
Hussain Gadelkarim Ahmed, 1,2 Mohmad \\ Ahmad Babikir Ibraheem Beely, ${ }^{3}$ Mohammed \\ Siddig Abd El Aziz, ${ }^{4}$ Fawaz D Alshammari, ${ }^{3}$ \\ Salah Eldin G Elzaki, ${ }^{6}$ Gamal Eldin Mohamed \\ Osman Elhussein,' Rashid Awad Abdalla \\ Salih,' Bassam Ahmed ALmutlaq,' Abdullah \\ Dakheel Alotaibi,' Majid Zannon Alturkstani ${ }^{7}$ \\ 'Department of Pathology, College of Medicine, University of \\ Hail, Saudi Arabia \\ 2Department of Histopathology and Cytology, FMLS, University \\ of Khartoum, Sudan \\ ${ }^{3}$ Department of Medical Laboratory, Faculty of Medicine, \\ University of Kordofan, Sudan \\ ${ }^{4}$ Department of Histopathology and Cytology, Faculty of \\ Medical Laboratory Science, Sudan University for Science and \\ Technology, Sudan \\ ${ }^{5}$ Department of Clinical Laboratory, College of Applied Medical \\ Science, University of Hail, KSA, Saudi Arabia \\ ${ }^{6}$ Department of epidemiology, Tropical Medicine Institute, Sudan \\ 'Batterjee Medical College, KSA, Saudi Arabia
}

Correspondence: Hussain Gadelkarim Ahmed, Department of Pathology, College of Medicine, 2440, University of Hail, Kingdom of Saudi Arabia, Email hussaingad1972@yahoo.com

Received: March 08, 2017 | Published: March 28, 2017

\section{Introduction}

Esophageal carcinoma is one of the most frequent malignant tumors in the world. ${ }^{1}$ Esophageal carcinoma is the sixth leading cause of cancer death in the world. ${ }^{2}$ Esophageal cancer comprises esophageal adenocarcinoma and squamous-cell carcinoma, which represent the core histological subtypes of esophageal cancer. ${ }^{3}$ Esophageal carcinoma characterized by its poor prognosis among gastrointestinal cancers. While esophageal squamous cell carcinoma dominates worldwide, Western nations have seen a marked rise in the incidence of esophageal adenocarcinoma that parallels the obesity epidemic. Efforts engaged toward early detection have been challenging, presumed that dysplasia and early cancer is generally asymptomatic. Nevertheless, significant progress has been made in the past 10 to 15 years that permit for endoscopic management and often cure in early stage esophageal cancer. ${ }^{4}$ Various factors have been implicated to the etiology of esophageal carcinoma. The carcinogenesis of esophageal adenocarcinoma is initiated by the acid exposure of the esophageal mucosa from stomach, ${ }^{5}$ whereas the esophageal squamous cell carcinoma is linked to the chronic irritation of carcinogens mainly by the cigarette smoking, ${ }^{6}$ alcohol ${ }^{7}$ and hot beverage. ${ }^{8}$ A potential role of HR-HPV in the rise of esophageal adenocarcinoma was proposed in recent times. Various meta-analyses support the consequence of a higher HR-HPV detection rate in regions associated with high risk for esophageal squamous cell carcinoma compared to low-risk areas. ${ }^{9}$ A meta-analysis displayed that HR-HPV16 and 18 can be detected in esophageal squamous cell carcinoma. HR-HPV16, but not HR-HPV18, is significantly associated with the risk of esophageal squamous cell carcinoma. ${ }^{10}$ Therefore, the aim of this study was to identify HR-HPV16 \&18 among Sudanese patients with esophageal cancer.

\section{Materials and methods}

This study examined malignant tissues obtained from 100 Sudanese patients with esophageal cancers. Of the 102 study subjects, 57 were males and 45 were females (male/female ratio, 1.26:1.00), aged between 21 and 98 years with mean age of 60 years old. All patients were previously diagnosed as having esophageal carcinomas. The diagnosis was based on clinical examination and histological features of the biopsy. Esophageal cancer diagnosis was confirmed base on Royal College of Pathologists criteria (Royal College of Pathologists, 2005). The esophageal cancer including 91/102 (89.2\%) squamous cell carcinomas (SCCs) and 11/102 (10.8\%) adenocarcinoma. All obtained tissue samples were tested for the presence of HR-HPV subtypes 16\&18 using Polymerase Chain Reaction (PCR) molecular technique. The sample included full coverage of patients with esophageal lesions referred to Radiation and isotope Centre Khartoum with available sample quantity and data.

\section{DNA extraction}

DNA was extracted adopting procedure described by Ahmed et al. ${ }^{13}$ DNA was extracted from paraffin-embedded samples, by immersing tissue section in xylene to dissolve the paraffin from the tissue, and then rehydrated using a series of ethanol washes. Proteins and harmful enzymes such as nucleases were digested by 
proteinase K. Buffer containing denaturing agent (sodium dodecyl sulfate (SDS)), was added to facilitate digestion. ${ }^{14}$ Nucleic acids were purified from the tissue lysate using buffer saturated phenol and high speed centrifugation. Following phenol extractions, RNase A was added to eliminate contaminating RNA. Additional phenol extractions following incubation with RNase A were used to remove any remaining enzyme. Sodium acetate and isopropanol were added to precipitate DNA, and high speed centrifugation was used to pellet the DNA and facilitate isopropanol removal. Washing with $70 \%$ ethanol was performed to remove excess salts, followed by centrifugation to re-pellet the DNA. ${ }^{15,16}$ DNA is re-suspended in distilled water, quantified and stored at $-20^{\circ} \mathrm{C}$ Purified DNA was subsequently used in downstream applications of PCR. $-20^{\circ} \mathrm{C}$ Purified DNA was subsequently used in downstream applications of PCR.

\section{DNA quantification}

To evaluate the DNA quantification after DNA extraction, we had analyzed DNA measurement using a NanoDrop spectrophotometer.

\section{Polymerase Chain Reaction (PCR) for detection of HPV}

PCR was applied to amplify the HPV E6/E7 gene of HPV 16 and 18 using type-specific primers (type-specific PCR, TS PCR). Below were primer sequences: HPV16 E6: forward 5'-CTG CAA GCA ACA GTT ACT GCG ACG-3', reverse 5'-CAT ACA TCG ACC GGT CCA CC-3', product of 315 bp; HPV 18 E7: forward 5'-GAG CCG AAC CAC AAC GTC AC-3', reverse 5'-GGA TGC ACA CCA CGG ACA CA-3', product of $152 \mathrm{bp}$.

\section{Gel-Electrophoresis}

The PCR products were visualized in $2 \%$ Agarose gel with $0.5 \mu \mathrm{g} /$ $\mathrm{ml}$ Ethidium bromide. Ten micro liters of $100 \mathrm{bp}$ DNA ladder and PCR product was loaded on the gel. Gel-electrophoresis was made at $120 \mathrm{~V}$ and $36 \mathrm{~mA}$ for 60 minutes. Images were reserved by Gel documentation system (Gel mega, digital camera and software in a computer)

\section{Ethical consent}

The study was approved by Faculty Research Board, Faculty of Medical Laboratory Science, Sudan University for Science and Technology and Technology \& Radiation and Isotope Hospital. This in addition to the fact that, the authors followed the tenants of the Declaration of Helsinki.

\section{Data analysis}

The data were analyzed for presence of HR-HPV16 in esophageal cancer tissues. Relative risks (RRs) and 95\% confidence intervals (95\% CIs) were calculated. Data management was done using Statistical Package for Social Sciences (SPSS version 16). SPSS was used for analysis and to perform Pearson Chi-square test for statistical significance ( $\mathrm{P}$ value). The 95\% confidence level and confidence intervals were used.

\section{Results}

In this study 102 tissue specimens were tested for the presence of HR-HPV subtypes $16 \& 18$. The samples were obtained from 57 males and 45 females their age ranging from 21 to 98 years old with a mean age of 60 years. The majority of the study populations were at the age range 66-75 years constituting 26/102(25.5\%) patients followed by age ranges, both $56-65$ and $<45$ years representing $24 / 102(23.5 \%)$ patients for each group. Moreover, 16/102(15.7\%) patients were identified among age group 46-55 years, hence only $10 / 102(9.8 \%)$ patients were found among age group $76+$ years, as indicated in Figure1. However, most of the patients were diagnosed as having Squamous cell carcinoma representing 91/102(89.2\%) followed by Adenocarcinoma representing 11/102(10.8\%). Of the 91 patients with Squamous cell carcinoma, 48/91(52.7\%) were males and $43 / 91(47.3 \%$ were females. Of the 11 patients with Adenocarcinoma 9/11(81.8\%) were males and $2 / 11(18.2 \%)$ were females.

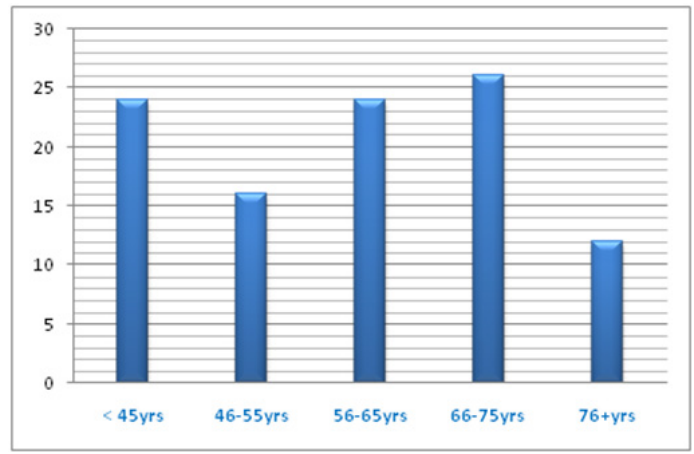

Figure I Description of the study population by age.

Regarding molecular detection of HR-HPV 16, positive findings were found in 25/102 (24.5\%) and couldn't be disclosed in $77(75.5 \%)$ of the study subjects, hence, none of the cases was identified with HPV18. Of the 57 males with esophageal cancer, 17/57 (29.8\%) were identified with HPV16 infection and the remaining 40/57(70.2\%) found without HPV16 infection. Of the 45 females with esophageal cancer, 8/45(17.8\%) were found positive for HPV 16 and the remaining 37/45(82.2 \%) were found negative for HPV16. According to cancer type, of the 91 cases of the Squamous cell carcinoma, 22/91 (24.2\%) were found with positive HPV16 and the remaining 69/91 $(75.8 \%)$ were negative. Of the 11 cases of the Adenocarcinoma, $3 / 11(27.3 \%)$ were found with positive HPV16 and the remaining 8/11 $(72.7 \%)$ were negative, as indicated in Table 1, Figure 2. With regard to HR-HPV16 with age distribution, the highest positive results were found in age group 66-75 years, representing $9 / 25(36 \%)$, followed by $56-65,<45$ and $46-55$ constituting $6 / 25(24 \%), 5 / 25(20 \%)$ and $4 / 25(16 \%)$ respectively, where's; the groups with the highest negative results were $<45$ years, representing $19 / 77(24.7 \%$ ) followed by $56-$ $65,66-75,46-55$ and $76+$ constituting 18/77(23.4\%), 16/77 (20.8\%), $13 / 77(16.9 \%)$ and $11 / 77(14 \%)$ in this order, as shown in Table 2 , Figure 3.

Table I Distribution of HR-HPV subtypes 16, by sex and cancer type

\begin{tabular}{llll}
\hline Sex & Positive & Negative & Total \\
\hline Males & 17 & 40 & 57 \\
Females & 8 & 37 & 45 \\
Total & 25 & 77 & 102 \\
Cancer type & & & \\
Squamous & 22 & 69 & 91 \\
Adenocarcinoma & 3 & 8 & 11 \\
Total & 25 & 77 & 102 \\
\hline
\end{tabular}

Table 2 Distribution of HPV 16 by age

\begin{tabular}{llll}
\hline Age group & Positive & Negative & Total \\
\hline$<45$ years & 5 & 19 & 24 \\
$46-55$ & 4 & 13 & 17 \\
$56-65$ & 6 & 18 & 24 \\
$66-75$ & 9 & 16 & 25 \\
$76+$ & 1 & 11 & 12 \\
Total & 25 & 77 & 102 \\
\hline
\end{tabular}




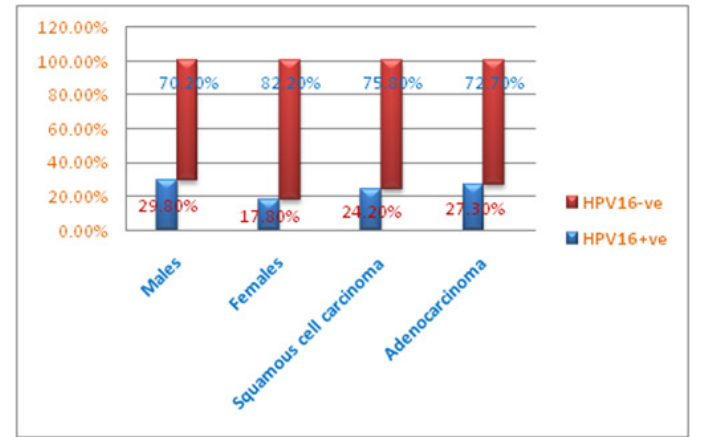

Figure 2 Description of HR-HPV subtypes 16, by sex and cancer type.

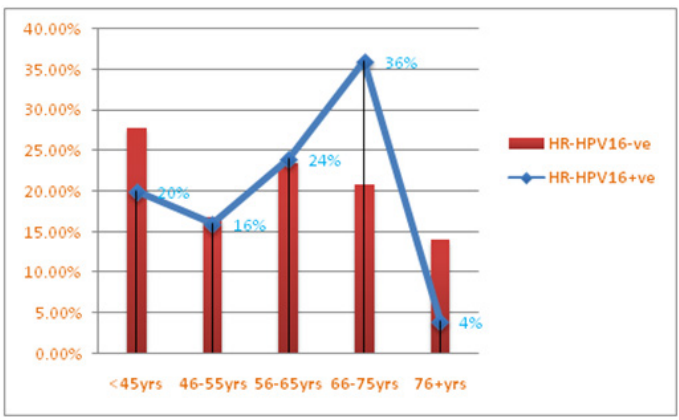

Figure 3 Description of HPVI6 by age.

\section{Discussion}

HPVs particularly HR-HPV subtypes 16 and 18 have been associated with the etiology of a number of cancers including cervical cancer, oral cancer and esophageal cancer. Although there is a rise in the incidence of esophageal cancer in Sudan, there is a lack of data regarding the relationship between HPV infection and esophageal carcinoma in the Sudan. Therefore the current study is one of the few studies attempt to explore the role of HPV in the etiology of esophageal cancer and to inspire further investigations in this field. The present study found a prevalence of $24.5 \%$ of HR-HPV16 infection among Sudanese patients with esophageal carcinoma. HRHPV related esophageal squamous cell carcinoma detection rates are significantly variable across different countries. Geographic location likely accounts for a majority of the variation in HPV prevalence, with high-incidence regions including Asia reporting significantly higher HR-HPV related esophageal squamous cell carcinoma infection rates compared with low-incidence regions such as Europe, North America, and Oceania. ${ }^{11}$ Studies have revealed that Asia is high-risk region. ${ }^{12-14}$ In a meta-analysis study, an overall HR-HPV prevalence of $30.6 \%$ was calculated, but a region-specific infection rate of $10.1 \%$ for Canada and the United States. ${ }^{12}$ There was evidence of an increased risk of esophageal squamous cell carcinoma in patients with HRHPV infection. The prevalence of HR-HPV in esophageal squamous cell carcinoma was found to be $24 \cdot 8 \%$. There was an increased risk associated with HPV-16 infection (OR 2·35, 95\% CI 1·73-3·19). Subgroup analyses indicated geographical inconsistency, with Asia (OR 2.94, 95\% CI 2·16-4·00), and particularly China (OR 2·85, 95\% CI 2.05-3.96) representing high-risk areas. ${ }^{15}$

However, the only one study we came across in the literature regarding the relationship between esophageal carcinoma and HR-HPV reported from Sudan, found a prevalence of $13 \%$ for HR-HPV16. ${ }^{16}$ In another study to determine the frequency and genotype of human papillomavirus (HPV) infections in head and neck squamous cell carcinomas (HNSCCs), 6 of the 150 (4\%) HNSCCs were HPV positive. HPV16 was the most prevalent type, with single infections present in 3/6 (50\%) cases, whereas HPV18 and HPV 33 were detected in 2/6 (33\%) and 1/6 (17\%), respectively. HPV infections were detected in $3(50 \%)$ cases of oral cavity and $3(50 \%)$ cases of pharynx. ${ }^{17}$ In the current study all of the detected cases of HR-HPV infections were infected with HR-HPV16. Such findings were reported in several studies. In a study investigated 8990 esophageal squamous cell carcinoma patients and 174 esophageal adenocarcinomas patients, the prevalence of HR-HPV in esophageal squamous cell carcinoma was $22.2 \%$, HR-HPV16 was the most frequently observed subtype with a summarized prevalence of $11.4 \%$. With respect to esophageal adenocarcinoma, HR-HPV prevalence was $35.0 \%$ (95\% CI, 13.2-65.7\%) and HR-HPV-16 prevalence was $11.4 \%$. Significant association was observed between HR-HPV infection and esophageal squamous cell carcinoma with a summarized odds ratio of 3.32 (95\% CI, 2.26-4.87). According to HR-HPV16, the strength of the association was found to be 3.52 (95\% CI, 2.04-6.07).$^{18}$ Though HR-HPV18 was not detected in the present study but studies have reported low prevalence rates in the highly infected regions. ${ }^{19}$

Although the prevalence of esophageal cancer was higher among males, but the prevalence rates of HR-HPV16 were relatively similar for both sex. However, we didn't come across study showing significant variation in the prevalence of HPV infections in regard to the sex. When calculating the prevalence rate from the same number of sex, it appears greater among females due to the low number of females in regard to the total number of the study population. In the present study we observed variable frequencies among different age ranges, but the HR-HPV infection was more frequent among elder population. The limitations in this study include; it's retrospective setting and the strict detection of only two HR-HPV subtypes. This in addition to the missing of some demographical data. But still it has a value in directing further studies in this context.

\section{Conclusion}

HR-HPV subtype 16 is significantly contributed to the etiology of esophageal cancer in Sudan. Further studies are required to determine the burden of other HR-HPV subtypes in esophageal cancer. Urgent preventive strategies are exceedingly recommended towards better control of esophageal cancer in Sudan.

\section{Acknowledgments}

None.

\section{Conflicts of interest}

None.

\section{References}

1. Tan C, Qian X, Guan Z, et al. Potential biomarkers for esophageal cancer. Springerplus. 2016;5:467.

2. Rustgi AK, El-Serag HB. Esophageal carcinoma. $N$ Engl J Med. 2014;371(26):2499-2509.

3. Rubenstein JH, Shaheen NJ. Epidemiology, Diagnosis, and Management of Esophageal Adenocarcinoma. Gastroenterology. 2015;149(2):302-317.e1.

4. Alsop BR, Sharma P. Esophageal Cancer. Gastroenterol Clin North Am. 2016;45(3):399-412.

5. Chung CS, Lee YC, Wu MS. Prevention strategies for esophageal cancer: Perspectives of the East vs. West. Best Pract Res Clin Gastroenterol. 2015;29(6):869-883. 
6. Kuang JJ, Jiang ZM, Chen YX, et al. Smoking Exposure and Survival of Patients with Esophagus Cancer: A Systematic Review and MetaAnalysis. Gastroenterol Res Pract. 2016(2016).

7. Thrift AP. The epidemic of oesophageal carcinoma: Where are we now? Cancer Epidemiol. 2016;41:88-95.

8. Andrici J, Eslick GD. Hot Food and Beverage Consumption and the Risk of Esophageal Cancer: A Meta-Analysis. Am J Prev Med. 2015;49(6):952-960.

9. Xu W, Liu Z, Bao Q, et al. Viruses, Other Pathogenic Microorganisms and Esophageal Cancer. Gastrointest Tumors. 2015;2(1):2-13.

10. Yong F, Xudong N, Lijie T. Human papillomavirus types 16 and 18 in esophagus squamous cell carcinoma: a meta-analysis. Ann Epidemiol. 2013;23(11):726-734.

11. Ludmir EB, Stephens SJ, Palta M, et al. Human papillomavirus tumor infection in esophageal squamous cell carcinoma. J Gastrointest Oncol. 2015;6(3):287-295.

12. Syrjänen K. Geographic origin is a significant determinant of human papillomavirus prevalence in oesophageal squamous cell carcinoma: systematic review and meta-analysis. Scand J Infect Dis. 2013;45(1):1-18

13. Yong F, Xudong N, Lijie T. Human papillomavirus types 16 and 18 in esophagus squamous cell carcinoma: a meta-analysis. Ann Epidemiol. 2013;23:726-734
14. Hardefeldt HA, Cox MR, Eslick GD. Association between human papillomavirus (HPV) and oesophageal squamous cell carcinoma: a meta-analysis. Epidemiol Infect. 2014142(6):1119-1137.

15. Hardefeldt HA, Cox MR, Eslick GD. Association between human papillomavirus (HPV) and oesophageal squamous cell carcinoma: a meta-analysis. Epidemiol Infect. 2014;142(6):1119-1137.

16. Ahmed HG, Beely MABI, Aziz MSAE, et al. Prevalence of high Risk Human Papilloma Viruses 16 and 18 (HRHPV16 \& 18) Among Sudanese Patients with Esophageal Cancer. J Cancer Prev Curr Res. 2016;5(6)

17. Ahmed HG, Mustafa SA, Eltom FM, et al. Frequency and genotype of human papillomavirus among Sudanese patients with head and neck tumors. Ecancer. 2012;6:282.

18. Li X, Gao C, Yang Y, et al. Systematic review with meta-analysis: the association between human papillomavirus infection and oesophageal cancer. Aliment Pharmacol Ther. 2014;39(3):270-281.

19. Liu T, Liu Q, Liang M, et al. Viral load of HPV 16/18 in esophageal squamous cell carcinoma in three ethnic groups living in Xinjiang Autonomous Region, China. Mol Biol Rep. 2013;40(2):2045-2052. 